\title{
Analysis of Einstein Field Equations of Static Plane Symmetric Space-Time in General Relativity via Lie Approach
}

\author{
Adil Jhangeer $^{1^{*}}$ \\ ${ }^{1}$ Department of Electrical Engineering, Namal Institute, Talagang Road, Mianwali 42250, Pakistan \\ ${ }^{*}$ Corresponding author
}

\author{
Article Info \\ Keywords: Lie algebra, Optimal sys- \\ tem, Plane symmetric solutions. \\ 2010 AMS: 35Qxx. \\ Received: 5 June 2018 \\ Accepted: 31 December 2018 \\ Available online: 20 April 2019
}

\begin{abstract}
In this study, Einstein field equations of static plane symmetric space-time is discussed by means of Lie approach. Lie point symmetries are computed for the considered equations. Moreover, one dimensional conjugacy classes for symmetry algebra are reported. Additionally, similarity variables are derived which are further utilized to find the solutions for parameters $A$ and $B$ of the considered space-time. Both linearly independent and dependent relationships between the unknown parameters $A$ and $B$ are observed for different cases. At the end, some well-known solutions are presented.
\end{abstract}

\section{Introduction}

It is now exactly 100 years since the birth of General Relativity (GR). Exact solutions of the Einstein field equations have played very important roles in the study of physical problems. Some well-known examples includes the Schwarzschild and Kerr solutions to discuss black holes, the Friedmann solutions in the context of cosmology and the plane wave solutions which ended the controversial discussion about the existence of gravitational radiation [1]. The Einstein field equations are highly non-linear second order partial differential equations (PDEs). On the other hand, Lie's theory gives a mathematical and systematic approach to investigate the solutions of differential equations. The application of Lie group theory for the solution of nonlinear ordinary differential equation is one of the most fascinating and significant area of research. It is well-established that from Lie's theory one can not only construct a class of exact solutions but can also find new solutions using different invariant transformations. It also gives most widely applicable technique to find the closed form solution of differential equations. Investigation of these solutions plays a vital role for the understanding of the physical aspects of these differential equations. Lie symmetry analysis is considered to be a systematic way to derive an ansatz which can be further utilized to reduce the dimension of the differential equations (DEs). The Lie analysis also plays a important role in the algebraic analysis of DEs [2]. Thus it would be worthwhile to investigate the solutions of Einstein field equations using Lie symmetry approach.

The space-time admitting three parameter group of motions of the Euclidean plane is said to possess plane symmetry and is known as a plane symmetric space-time. Such space-time possesses many properties equivalent to those of spherical symmetry. The plane symmetric space-time has been extensively investigated by many researchers from various standpoints. Taub [3], Bondi [4], Bondi and Pirani-Robinson [5] defined and studied plane wave solutions. They considered the concept of group of motions of space-time which played a fundamental role in the plane gravitational waves. It has been established that the plane symmetric space-time admits the plane wave solutions of GR field equations [6].

Here the aim is to explore the similarity solutions of static plane symmetric vacuum solutions in GR. For this purpose, Lie point symmetry method is used. In particular, exact solutions of field equations are presented. The paper is organized as follow: Lie analysis of plane symmetric vacuum solutions is given in Section 2. In the last section, results are summarized.

\section{Lie analysis for plane symmetric vacuum solutions}

Let us consider static plane symmetric space-time [7]:

$$
d s^{2}=A(x) d t^{2}-d x^{2}-B(x)\left(d y^{2}+d z^{2}\right) .
$$


The Ricci scalar for this space-time is given by

$$
R=\frac{1}{2}\left[\frac{2 A^{\prime \prime}}{A}-\left(\frac{A^{\prime}}{A}\right)^{2}+\frac{2 A^{\prime} B^{\prime}}{A B}+\frac{4 B^{\prime \prime}}{B}-\left(\frac{B^{\prime}}{B}\right)^{2}\right],
$$

where prime represents derivative with respect to $x$.

The Einstein field equations are:

$$
R_{\mu \nu}-\frac{1}{2} R g_{\mu \nu}=\frac{8 \pi G}{c^{4}} T_{\mu \nu} .
$$

Using Eq.(2.1), GR vacuum field equations becomes:

$$
\begin{aligned}
\frac{4 B^{\prime \prime}}{B}-\left(\frac{B^{\prime}}{B}\right)^{2} & =0, \\
\left(\frac{B^{\prime}}{B}\right)^{2}+\frac{2 A^{\prime} B^{\prime}}{A B} & =0, \\
\frac{2 A^{\prime \prime}}{A}+\frac{2 B^{\prime \prime}}{B}-\left(\frac{A^{\prime}}{A}\right)^{2}-\left(\frac{B^{\prime}}{B}\right)^{2}+\frac{A^{\prime} B^{\prime}}{A B} & =0 .
\end{aligned}
$$

Manipulating Eqs.(2.2-2.4), one can get:

$$
\frac{A^{\prime} B^{\prime}}{A B}+\left(\frac{B^{\prime}}{B}\right)^{2}-\frac{2 B^{\prime \prime}}{B}=0, \quad \frac{2 A^{\prime \prime}}{A}-\left(\frac{A^{\prime}}{A}\right)^{2}+\frac{A^{\prime} B^{\prime}}{A B}-\frac{2 B^{\prime \prime}}{B}=0 .
$$

Thus a system of second order non-linear differential equations with two unknowns namely $A$ and $B$ is obtained.

The Lie analysis of (2.5) requires one parameter Lie group of transformations:

$$
\begin{aligned}
\tilde{x} & =x+\lambda \xi(x, A, B)+O\left(\lambda^{2}\right), \\
\tilde{A} & =A+\lambda \phi(x, A, B)+O\left(\lambda^{2}\right), \\
\tilde{B} & =B+\lambda \psi(x, A, B)+O\left(\lambda^{2}\right),
\end{aligned}
$$

which leave the solutions of differential equation invariant.

Let

$$
X \equiv \xi(x, A, B) \frac{\partial}{\partial x}+\phi(x, A, B) \frac{\partial}{\partial A}+\psi(x, A, B) \frac{\partial}{\partial B}
$$

be a Lie point symmetry generator of (2.5) if it satisfies the invariance condition i.e.

$$
\left.X^{[2]}[E q \cdot(2.5)]\right|_{E q \cdot(2.5)}=0,
$$

where $X^{[2]}$ is the second prolongation [2].

From Eqs. (2.6)-(2.8), one can write:

$$
\frac{d x}{\xi(x, A, B)}=\frac{d A}{\phi(x, A, B)}=\frac{d B}{\psi(x, A, B)}=\lambda
$$

which is known as the characteristics equation [2]. Using Eq. (2.9) one can get an over determined system of linear partial differential equations which yields:

$$
\xi=c_{1} x+c_{2}, \quad \phi=c_{3} A, \quad \psi=c_{4} B,
$$

where $c_{i}(i=1,2,3,4)$ are the constants of integration. Now Lie point symmetries for the system (2.5) are constructed by setting one constants equal to unity and remaining constants to zero in (2.10):

$$
X_{1}=x \frac{\partial}{\partial x}, X_{2}=\frac{\partial}{\partial x}, X_{3}=A \frac{\partial}{\partial A}, X_{4}=B \frac{\partial}{\partial B} .
$$

\subsection{Optimal system}

The finite-dimensional Lie algebra of the system (2.5) is $L=\left\{X_{1}, X_{2}, X_{3}, X_{4}\right\}$. As we know there are infinitely many one-dimensional Lie algebras. The next aim is to obtain a class from these infinitely many one-dimensional Lie algebras which is their best representative. This class is called the optimal system of one-dimensional algebras.

In order to construct the conjugacy classes for one-dimensional algebra, following definitions from [2] are presented.

1: If $X_{i}$ and $X_{j}$ are Lie point generators then their Lie bracket or commutator $\left[X_{i}, X_{j}\right]$ is defined in a unique way, satisfying:

$$
\left[X_{i}, X_{j}\right]=X_{j}\left(X_{i}\right)-X_{i}\left(X_{j}\right)
$$


The nonzero commutation relationship for $L$ is:

$$
\left[X_{1}, X_{2}\right]=X_{2}
$$

2: $£_{i}$ and $£_{j}$ are said to be equivalent conjugacy classes if

$$
£_{i}=\operatorname{Ad} X_{i}\left(£_{j}\right)
$$

where $X_{i} \in L$.

The general element will be

$$
£=a_{1} X_{1}+a_{2} X_{2}+a_{3} X_{3}+a_{4} X_{4} .
$$

After applying adjoint transformation on $£$ and renaming the coefficients, one gets [8]:

$£_{1}=<X_{2}>, \quad £_{2}=<a X_{3}+b X_{4}>, \quad £_{3}=<X_{2}+\varepsilon\left(a X_{3}+b X_{4}\right)>, \quad £_{4}=<X_{1}+\alpha\left(a X_{3}+b X_{4}\right)>$,

where $a=\cos \theta, b=\sin \theta$ and $\theta \in[0, \pi)$. While $\alpha$ is a real number and $\varepsilon= \pm 1$.

\subsection{Reduction by calculating similarity variables}

This section is devoted for the computation of similarity variables and their corresponding reduced equations.

2.2.1. $£_{1}=<X_{2}>$

Using Eq.(2.10), the characteristic system becomes:

$$
\frac{d x}{1}=\frac{d A}{0}=\frac{d B}{0}
$$

which can be separated into the following linear equations:

$$
\text { (i) : } \frac{d A}{0}=\frac{d x}{1} \quad(i i): \frac{d x}{1}=\frac{d B}{0} .
$$

It leads towards a constant solution.

2.2.2. $£_{2}=<a X_{3}+b X_{4}>$

I. $a=1, b=0$.

It gives:

$$
X_{3}=A \frac{\partial}{\partial A} .
$$

Using Eq.(2.10), this operator has the following characteristic system:

$$
\frac{d A}{A}=\frac{d x}{0}=\frac{d B}{0}
$$

which can be separated into the following linear equations:

$$
\text { (i) }: \frac{d A}{A}=\frac{d x}{0} \quad(i i): \frac{d A}{A}=\frac{d B}{0} .
$$

Integrating $((i)-(i i))$, one by one we get the following results respectively:

$$
x=\xi_{1}, \quad B=\xi_{2},
$$

where $\xi_{1}$ and $\xi_{2}$ are constants of integration. Designating second invariant as a function of first one gives:

$$
B=g\left(\xi_{1}\right),
$$

where $A$ is a constant function. Using $B=g\left(\xi_{1}\right)$ in the system (2.5), one can obtain $A=B=$ constant, leaving a Minkowskian space-time.

\section{II. $a=0, b=1, \quad$ (Linearly independent relation)}

Similarly,

$$
x=\xi_{1}, \quad A=\xi_{2} .
$$

Designating second invariant as a function of first one it yields: $A=g\left(\xi_{1}\right)$ and $B$ is a constant function. System $(2.5)$ with $A=g\left(\xi_{1}\right)$ gives the following solution

$$
A=C_{3}\left(x-2 C_{2}\right)^{2}, B=C_{1},
$$

where $C_{i}$ 's are real constants. By some suitable substitutions, it can be shown that this solution corresponds to conformally flat space-time. 


\section{Concluding remarks}

This paper is devoted to investigate the plane symmetric solutions in GR. Here the Lie point symmetry approach has been used to investigate the plane symmetric solutions. When infinitesimal generator is applied to the system (2.5), an over-determined system of linear partial differential equations is obtained. Thus solution of this system gives four non-trivial Lie point generators, which are further utilized to compute the one-dimensional optimal system. Different cases are generated from these classes which in turn suggest both linearly independence and dependence relationships between the parameters $A$ and $B$ for plane symmetric space-time in GR.

It is expected that this approach may recover some new solutions. It would be worthwhile to extend the analysis to explore the solutions for other space-times. In particular, the solutions of field equations in modified theories of gravity can be interested to discuss the issue of dark energy and expansion of universe. Investigation of non-vacuum plane symmetric solutions in $f(R)$ gravity using Lie approach is under process.

\section{References}

[1] D. Kramer, H. Stephani, M. MacCullum, E. Herlt, Exact Solutions of Einstein Field Equations, Cambridge University Press, 1980.

[2] P. J. Olver, Applications of Lie Groups to Differential Equations, Springer-Verlag, New York, 1986.

[3] A. H. Taub, Empty space-times admitting a three parameter group of motions, Annals Math., 53 (1951), 472-490.

[4] H. Bondi, Plane gravitational waves in general relativity, Nature, 179 (1957), 1072-1073.

[5] H. Bondi, E. Pirani, I. Robinson, Gravitational waves in general relativity. III. exact plane waves, Proc. Roy. Soc., 251 (1959), $519-533$.

[6] H. Takeno, On plane wave solution of field equations in general relativity, N. S. Tensor, 7 (1957), 97-102.

[7] M. Sharif, M. F. Shamir, Plane symmetric solutions in $f(R)$ gravity, Mod. Phys. Lett., A25 (2010), $1281-1288$.

[8] J. Patera, P. Winternitz, Subalgebras of real three- and four-dimensional Lie algebras, J. Math. Phy., 18(7) (1977), 1449-1455. 